\title{
EDITORIAL
}

\section{Ultrasound Use in Ski Clinics}

Ultrasonography is becoming more commonplace in various emergency settings. In this issue of Wilderness \& Environmental Medicine, the article by Evans and Harris, ${ }^{1}$ entitled "Ultrasound and Ski Resort Clinics: Mapping Out the Potential Benefits," clearly points out several areas of potential benefit to clinicians who practice in ski clinics. As ultrasonography specialists practicing in a locale with many ski clinics, we agree that the incorporation of ultrasonography should strongly be considered by those who practice in such settings. There are also a number of limitations to this technology, and an immediate investment in it may not practical for all ski clinics.

One consideration should be the type of practitioner staffing at the clinic and the likelihood of universal use by all practitioners. Ski clinics are staffed by a wide variety of practitioners from middle level and nurses to physicians of various specialties including but not limited to emergency medicine, orthopedics, general surgery, family practice, and pediatrics. Additionally, during several hours per week, many clinics are staffed by midlevel practitioners or nurses only. Staffing of clinics with physicians trained in point-of-care ultrasonography is an important consideration. Ultrasonography is now a requirement for emergency medicine training. However, the majority of physicians who currently practice medicine around the country have been out of residency training for years and graduated before implementation of these new training requirements. It is only recently that point-of-care ultrasonography has gained wide acceptance and has become a core competency in emergency medicine training programs. And although emergency medicine training programs have clearly been the progressive and early adapters of this technology, it will likely take many more years before the majority of practicing emergency medicine physicians are adequately trained in this technology.

The majority of other medical training specialty programs (eg, family practice, internal medicine) have not adapted this technology as readily as their emergency medicine colleagues. As a result, the majority of these physicians have very little exposure to ultrasound technology during their training. Because many ski clinic physicians are not trained in emergency medicine, it may prove difficult for the majority of physicians to use ultrasound equipment at the bedside and feel confident in their ability to obtain adequate images and to make treatment and triage decisions based on those images. Sensitivity and specificity are greatly affected by the quality of the images obtained and by operator competency with ultrasound examinations. ${ }^{2}$ Lack of training can lead to low confidence with scan interpretations and ultimately discourage the use of this technology. As a solution to this potential problem, the Evans and Harris ${ }^{1}$ discuss the use of telemedicine and specially developed training programs that have proved effective in training novice physicians to use ultrasound equipment. Although these models are proven and effective in the short term, however, they take a strong continual commitment within the physician group and technological infrastructure to develop and maintain.

Problems that may arise with archiving of images as well as documentation of ultrasonograms for charting purposes is another potential barrier to the technical implementation of ultrasonography. Traditionally, a director of ultrasonography is given this responsibility and some level of compensation is negotiated to spearhead these issues. Certainly, if clinicians are making medical decisions based on image feedback, then proper archiving of these images should take place. Meeting quality assurance guidelines may prove to be difficult if there is not someone already trained and willing to review scans and provide feedback to the physician group.

Purchasing newer portable ultrasound equipment typical of that used in emergency departments in the United States is generally in the range of $\$ 30000$ to $\$ 60000$, depending on application needs and probe configuration. Although ultrasound systems can be purchased in the $\$ 10000$ price range, these systems would typically be refurbished ultrasound equipment that has years of prior use and aging hardware. Considerations when purchasing refurbished ultrasound machines would be the lifespan and durability of the hardware along with the stability of the operating system and user interface. Additionally, only limited single year warranties are typically available with those types of purchases.

Small handheld ultrasound devices have recently made their appearance on the market and would prove to 
be a perfect fit for a mountain portal and ski clinic setting. However, it is also our experience that these devices have low processing power and image quality, making their use in the clinical setting extremely limited. Clinics across the United States vary in funding, but we assume that many may not be able to budget for reasonably priced ultrasound equipment that meets the clinical needs and assures long-term stability and use in mountain patrol and ski clinic settings.

Ultrasonography has been used to diagnose certain orthopedic ski injuries such as long bone fractures, but although it has been proven to have high sensitivity and specificity for these fractures, it has not been proven superior to radiography. ${ }^{3}$ Many US ski clinics have access to radiography equipment, and the addition of ultrasonography may not add to the orthopedic diagnostic capabilities in these clinics. It would likely prove useful in more rural settings and in clinics without radiography available to aid in fracture diagnosis.

Some of the most common injuries listed in the article were wrist and thumb injuries, which we have found to be difficult to assess even for advanced users of ultrasonography. Currently, most studies validating ultrasonograms for fractures have been done on long bones, showing higher accuracy with midshaft fractures.

Shoulder dislocations can be diagnosed and the reductions verified quickly using ultrasonography, thus potentially eliminating the need for before and after films. ${ }^{4}$ These techniques are not particularly difficult to learn and may speed patient times by not needing to wait for before and after reduction films. Using ultrasonograms only (without using radiographs) for diagnosis and treatment of shoulder dislocations has been criticized because it has not been prospectively validated. The practitioner may miss concomitant fractures of the humerus. It has been shown to be quite accurate in patients with recurrent dislocation, and who are at lower risk for fracture due to mechanism. ${ }^{5}$ Depending on the pre-test probability and the patient's history, using an ultrasound-only approach could greatly speed the time to treatment as well as decrease cost incurred by the patient. This method does, however, increase the risk of missing fractures.

Spinal column injuries are, unfortunately, seen commonly in the ski clinic setting. Floyd ${ }^{6}$ cited a rate of 1 injury per 100000 skier and snowboarder days for spinal column injuries. Although there is been a small amount of literature showing the utility of using ultrasonography to define unstable vertebral burst fractures, ${ }^{7}$ these studies were performed on patients with chronic injuries. The risks of positioning a patient correctly to find these fractures, as well as the extremely high liability of missing these fractures, makes using ultrasonography in this setting unpractical. If suspicion is high enough for an unstable vertebral fracture, the best management would be stabilization on a backboard taking full cervical spine precautions for transfer and avoiding further manipulation while readying the patient for a quick transfer to where definitive imaging (radiography or computed tomography scan) could be done.

It was stated in the study by Evans and Harris ${ }^{1}$ that ultrasonography may prove useful in the diagnosis and management of the patient with head injury in the ski clinic setting. Recent articles have suggested that a diameter greater than $5 \mathrm{~mm}$ on optic nerve sheath diameter (ONSD) measurement is highly sensitive for increased intracranial pressure. Typically, however, it is the patient with more severe head injury who presents with increased intracranial pressure. The majority of severely head injured patients present with signs and symptoms that make it clinically apparent to the treating physician that the patient needs to be transferred to a higher level of care. In the study by Tayal et al, ${ }^{8} 7$ of 8 patients who had increased intracranial pressure had Glasgow Coma Scale scores of 14 or less. Unfortunately, when measuring ONSD for any type of intracranial injury, sensitivity proved less. Ultrasonography for ONSD greater than 5 $\mathrm{mm}$ had a $83 \%$ sensitivity for any intracranial injury and a specificity of $73 \%$. The potential problem of using ultrasonography to make decisions about transferring patients with reported head injury is that the positive predictive value of the test is low (30\%), and that may ultimately result in physicians unnecessarily transferring patients with no injury who they would have otherwise have observed on the mountain.

Nerve blocks using landmark techniques have been used for years in emergency settings, often for procedures. The use of ultrasonograms for block placement has increased the success rate, and also has been shown to decrease the complication rates, including nerve and vascular injury. ${ }^{9}$ Patients with painful orthopedic injuries are often undertreated for pain and undergo long transport times. This hesitancy to treat pain may stem from concern for deterioration during transportation, particularly in the elderly and in head trauma cases. Nerve blocks are an alternative to narcotic pain control without increasing concern for airway issues. Providers practicing in more rural settings with longer transport times could benefit their patients by being familiar with ultrasound-guided nerve blocks to greatly aid in pain relief during transport.

The focused assessment for trauma examination is considered commonplace in the workup of blunt abdominal trauma and has been shown to improve time to definitive treatment and outcomes. ${ }^{10}$ The extended focused assessment for trauma examination also includes evaluation of the pleural cavity. The ski clinic patient 
population has a high incidence of blunt trauma and could benefit from adjuncts to the trauma evaluation, including bedside ultrasonography, which has become standard of care in larger facilities. Many clinics do not have access to more expensive imaging modalities such as computed tomography, and having a bedside modality that could quickly evaluate free intraperitoneal fluid could lead to faster transport time to definitive management as opposed to long observation with serial examinations, which could affect outcomes when transfer delays are taken into account.

Pulmonary examinations also are quickly and easily done during the initial workup of trauma to evaluate the patient for pneumothorax and hemothorax. ${ }^{11}$ Ultrasonography has been shown to be more sensitive than chest plain X-ray film in evaluation of pneumothorax. ${ }^{12}$ Treatment decisions about thoracostomy tubes could also be made more rapidly with better information after a bedside assessment. Knowing there is a pneumothorax even in a stable patient may change the decision to place a thoracostomy tube based on time of transport or type of transport, air or ground, to prevent deterioration en route to a higher level of care. The extended focused assessment for trauma examinations are already widely accepted ideas in more traditional emergency settings and are being included in competency training around the country, making them a practical adjunct in the ski clinic setting.

We agree there are many potentially uses for ultrasonography at a ski clinic. However, that potential has to be weighed carefully against the capital cost and likelihood of use by the physicians who practice at each clinic. As ultrasonography becomes more universally available in training programs and more readily used by all physicians, its incorporation by ski clinics will be more meaningful.

Matthew Ahern, DO Angela McKellar, MD

Division of Emergency Medicine University of Utah Salt Lake City, UT

\section{References}

1. Evans CS, Harris NS. Ultrasound and ski resort clinics: mapping out the potential benefits. Wilderness \& Environ Med. 2012;23:239-247.

2. Ma OJ, Mateer JR. Prospective analysis of a rapid trauma ultrasound examination performed by emergency physicians. J Trauma. 1995;38:879-885.

3. Patel DD, Blumberg SM, Crain EF. The utility of bedside ultrasonography in identifying fractures and guiding fracture reduction in children. Pediatr Emerg Care. 2009;25: 221-225.

4. Blakeley CJ, Spencer Newman-Saundert O, Hahemi K. A novel use of the portable ultrasound in the management of shoulder dislocation. Emerg Med J. 2009;26:662-663.

5. Hendey GW. Necessity of radiographs in the emergency department management of shoulder dislocations. Ann Emerg Med. 2000;36:108-113.

6. Floyd T. Alpine skiing, snowboarding, and spinal trauma. Arch Orthop Trauma Surg. 2001;121:433-436.

7. Vordemvenne T, Hartensuer R, Lohrer L, Vieth V, Fuchs $\mathrm{T}$, Raschke MJ. Is there a way to diagnose spinal instability in acute burst fractures by performing ultrasound? Eur Spine J. 2009;18:964-971.

8. Tayal VS, Neulander M, Norton HJ, Foster T, Saunders T, Blalvas M. Emergency department sonographic measurement of optic nerve sheath diameter to detect findings of increased intracranial pressure in adult head injury patients. Ann Emerg Med. 2007;49:508-514.

9. Bhoi S, Sina TP, Rodha M, Bhasin A, Ramchandani R, Galwankar S. Feasibility and safety of ultrasoundguided nerve block for management of limb injuries by emergency care physicians. J Emerg Trauma Shock. 2012;5:28-32.

10. Branney SW, Moore EE, Cantril SV, Burch JM, Terry SJ. Ultrasound based key clinical pathway reduces the use of hospital resources for the evaluation of blunt abdominal trauma. J Trauma. 1997;42:1086-1090.

11. Ma OJ, Mateer JR. Trauma ultrasound examination versus chest radiography in the detection of hemothorax. Ann Emerg Med. 1997;29:312-316.

12. Nagarsheth K, Kurek S. Ultrasound detection of pneumothorax compared with chest X-ray and computed tomography scan. Am Surg. 2011;77:480-484. 